Z. Klin. Chem. Klin. Biochem.

11. Jg. 1973 , S. $548-552$

\title{
Immunologische Diagnostik der IgA-Paraproteine
}

\author{
Von HILDE GöTZ \\ Forschungslaboratorium für Transplantations- und Tumorimmunologie der Chirurgischen Universitätsklinik \\ (Direktor: Prof. Dr. E. S. Biicherl) im Klinikum Westend der Freien Universität Berlin
}

(Eingegangen am 24. Februar 1972/September 1973)

122 Seren mit IgA-Paraproteinämie wurden aufgrund der immunelektrophoretischen Position ibres Paraproteins differenziert. Dabei konnten fünf verschiedene IgA-Paraprotein-Typen beobachtet werden: in $\alpha_{2}-, \alpha_{2} / \beta-, \beta-, \beta / \gamma_{1^{-}}$-sowie in $\gamma_{1}$-Stellung. Sie wurden mit IgA-I, IgA-II, IgA-III, IgA-IV und IgA-V bezeichnet. Am häufigsten vertreten war der Typ IgA-IV, es folgten der Typ IgA-III und der Typ IgA-V. Am seltensten wurde der Typ IgA-II beobachtet. Es handelt sich bei den verschiedenen IgA-Typen um einen elektrophoretischen Polymorphismus, bei welchem möglicherweise genetische Faktoren eine Rolle spielen. Den Befunden sollte vor allem bei der elektrophoretischen und immunelektrophoretischen Diagnostik Beachtung geschenkt werden.

\section{Immunological diagnosis of IgA-paraproteins}

122 Sera with IgA-paraproteinaemia were differentiated on the basis of the immunoelectrophoretic position of their paraprotein. Five different IgA-paraprotein types were observed, corresponding to positions $\alpha_{2}, \alpha_{2} / \beta, \beta, \beta / \gamma_{1}$, and $\gamma_{1}$. They were designated IgA-I, IgA-II, IgA-III, IgA-IV and IgA-V. Type IgA-IV occurred most frequently, followed by type IgA-III and type IgA-V. The rarest was type IgA-II. The differences between the various IgA-types are the result of an electrophoretic polymorphism, which is possibly genetically determined. These findings should be taken into account, especially in electrophoretic diagnosis.

Unter 640 immunelektrophoretisch analysierten Seren mit Paraproteinämien wurden 122 IgA-Paraproteine aufgedeckt. Aufgrund ihrer unterschiedlichen Beweglichkeit im elektrophoretischen Feld konnten mehrere Subtypen differenziert werden, welche im $\alpha_{2^{-}}$, im $\beta$ sowie im $\gamma$-Globulin-Bereich zur Darstellung gelangten.

Erfahrungsgemäß kann die immunologische Diagnostik, vor allem der in $\alpha_{2}$ - oder $\beta_{1}$-Stellung gelegenen IgAParaproteine, gewisse Schwierigkeiten bereiten. Es sollen deshalb im nachfolgenden einige praktische Richtlinien angeführt werden, bei deren Berücksichtigung ein Übersehen, etwa eines seltenen IgA-Paraproteins in $a_{2}$-Position, oder eine Fehlinterpretation ausgeschlossen werden können.

\section{Methoden}

Einfache Elektrophorese auf Celluloseacetatfolien (1) oder auf Papier (2)

Es empfiehlt sich, vor jeder Immunelektrophorese und vor jeder quantitativen immunologischen Plasmaproteinbestimmung die einfache Elektrophorese auf Celluloseacetatfolien oder Papier vorzunehmen. Dabei eignet sich der gefärbte Streifen zur Erkennung eines M-Gradienten mindestens ebenso gut wie das Diagramm. Die bloßen Relativ- oder Absolut-Prozente der einzelnen Fraktionen sind für diese Frage nicht ausschlaggebend. Dies gilt insbesondere für die Erkennung diskreter, schmaler Zwischenfraktionen, die häufig genug bei der Berechnung zu einer der benachbarten physiologischen Fraktionen geschlagen und damit nicht berücksichtigt werden.

Bei jeder auffällig schmal und zudem verstärkt dargestellten Fraktion im $\gamma-, \beta-, \alpha_{2}$-Globulin- und sogar im Albumin-Bereich ${ }^{1}$ ) sowie bei Komponenten, die in Bereichen zwischen physiologischen Proteingruppen in Erscheinung treten (Abb. 1), muß die immunelektrophoretische Differenzierung unter Verwendung eines

1) Verbreiterte Albuminfraktion oder „Bisalbuminämie“!

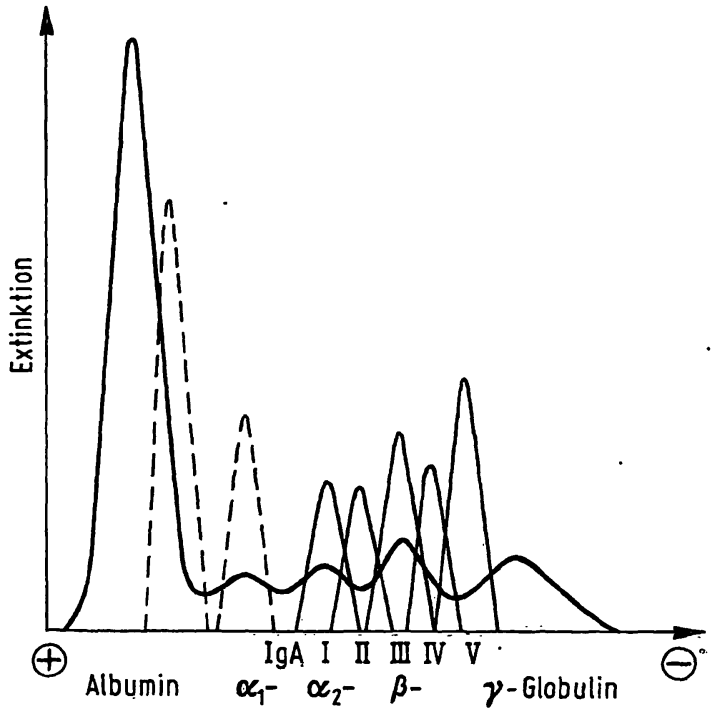

Abb. 1

IgA-Paraprotein-Stellungen nach einfacher Elektrophorese auf Celluloseacetatfolie (oder Papier). Zusammengedrängte, halbschematische Darstellung

Die gestrichelten Paraproteingradienten stammen nicht aus dem eigenen Beobachtungsgut, sondern aus. Angaben der einschlägigen Literatur

polyvalenten anti-Humanserums sowie monovalenter Antiseren gegen die verschiedenen Immunglobulin-Klassen veranlaßt werden. Darüber hinaus sollten die zweidimensionale DoppelImmundiffusion zur Auffindung der Äquivalenzzonen sowie die einfache, radiale Immundiffusions-Technik zur quantitativen Erfassung der IgA-Paraproteinkonzentration wie auch der Konzentrationen der übrigen Immunglobuline zur Anwendung kommen.

Immunelektrophorese $(3,4)$

Paraproteine sind, ungeachtet ihrer elektrophoretischen Position, Immunglobuline (Abb. 2 u. 3). Die immunelektrophoretische Analyse dient deshalb im wesentlichen der Zuordnung eines $M$ Gradienten zu einer der bekannten Immunglobulin-Klassen. Dabei sollte folgendermaßen vorgegangen wexden: 


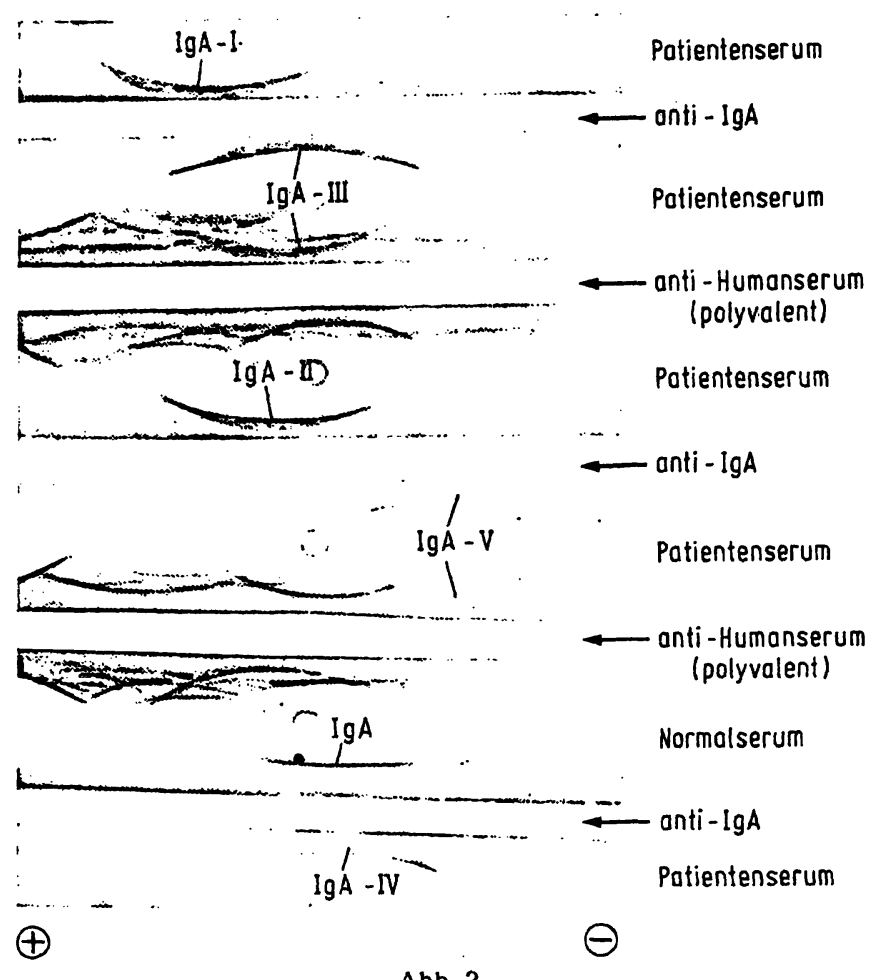

Abb. 2

Immunelektrophoretische Befunde elektrophoretisch unterschiedlich wandernder IgA-Paraproteine

Die Seren waren wegen der jeweils starken IgA-Paraproteinkonzentration in diesem Ansatz $1: 2$ bzw. $1: 6$ mit physiol. NaCl-Lösung verdünnt worden

Aufgrund der Farbintensität der Paraprotein-verdächtigen Komponente wird zunächst geschätzt, in welcher Konzentration bzw. Verdünnung das Patientenserum untersucht werden soll. Bei hoher Konzentration der M-Komponente kommt es zu unerwünschten Antigenüberschußphänomenen, welche u. U. eine Beurteilung des Präzipitats unmöglich machen. In solchen Fällen muß deshalb das Patientenserum verdünnt werden. Da man nie ganz sicher sein kann, welche der geschätzten Verdünnung für die immunelektrophoretische Differenzierung optimal ist, wird man am besten mindestens zwei verschiedene Verdünnungsgrade, z. B. $1: 6$, sowie $1: 12$, einsetzen.

Die Immunelektrophorese wird als Analysensatz vorgenommen, wobei sich Agarplatten mit den Abmessungen von $7,5 \times 9,0 \mathrm{~cm}$ am besten eignen. Es sollten grundsätzlich für jedes Serum zwei dieser Platten vorbereitet werden. Agarkonzentration, Pufferzusammensetzung (des Agar- und des Elektrodenpuffers) sowic Wanderungs- und Diffusionsbedingungen entsprechen den hinlänglich bekannten $\Lambda$ ngaben der Immunelektrophorese-Technik. Es ändert sich lediglich die für eine Platte benötigte Agarmenge, sie entspricht $10 \mathrm{ml}$. Jede dicser Platten nimmt 7 Einzelanalysen (= Auftragestellen) und 6 Antiserum-Kanäle auf. Dic Maße eines Einzelansatzes entsprechen den Maßen der Objektträget-Technik.

Jede der beiden vorbereiteten Platten wird mit zwei verschicdenen Verdünnungen desselben Patientenserums alternativ beschickt. Für die erste Platte kommen an Antiseren ${ }^{2}$ ) folgende zur Anwendung:

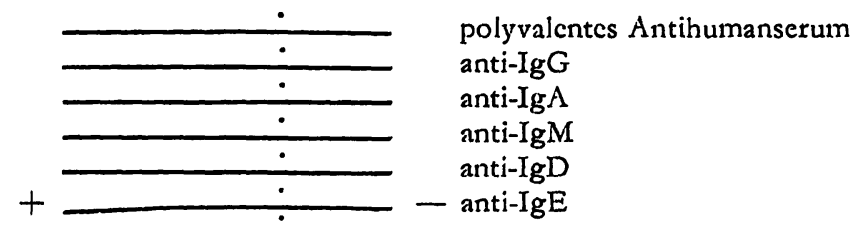

Für dic zweite Platte kommen folgende Antiseren in Frage:

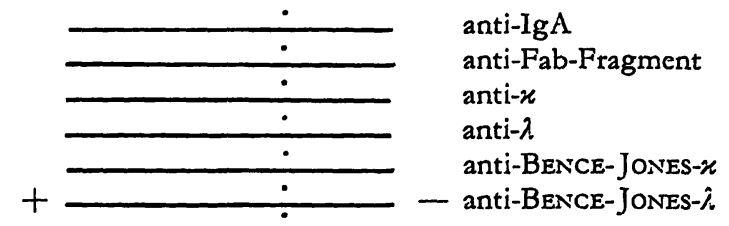

Die Åquivalenzzonen sollten mit Hilfc der ImmundiffusionsTechnik nach OuckrterionY (5) aufgeklärt werden (s. u.).

Zum Ausschluß bzw. zur Auffindung einer BenCe-Jones-Proteinämie und -Protcinurie muß unter allen Umständen der Urin des Patienten nach dem Schema der Platte 2 mituntersucht werden. Eine vorherige Gesamteiweiß-Bestimmung wird darüber entscheiden, ob der Urin eingeengt werden muß oder nicht.

Bei der Beurteilung der immunelektrophoretischen Befunde ist ganz allgemein auf Antigenüberschußphänomene, Längsspaltungen, Spornbildungen sowie auf Verformungen in sich einheitlicher Präzipitate zu achten. Beweisend für IgA-Paraprotein sind im wesentlichen die spezifische Immunpräzipitatbildung mit einem anti-IgA-Serum, sowie ein umschriebenes (monoclonales) Antigenüberschußphänomen des IgA-spezifischen Präzipitatbogens. Bei IgA-Paraproteinen in $\alpha_{2}$-Stellung findet sich häufig darüber hinaus eine Art „Kometenschweif“", d. h. eine unscharf

2) Behringwerke AG, 3550 Marburg/Lahn; Fresenius KG, $6380 \mathrm{Bad}$ Homburg v. d. H.; Hyland/Travenol International, 8000 München; Dacopatts $\mathrm{A} / \mathrm{S} / \mathrm{M}$ edac, 2000 Hamburg.
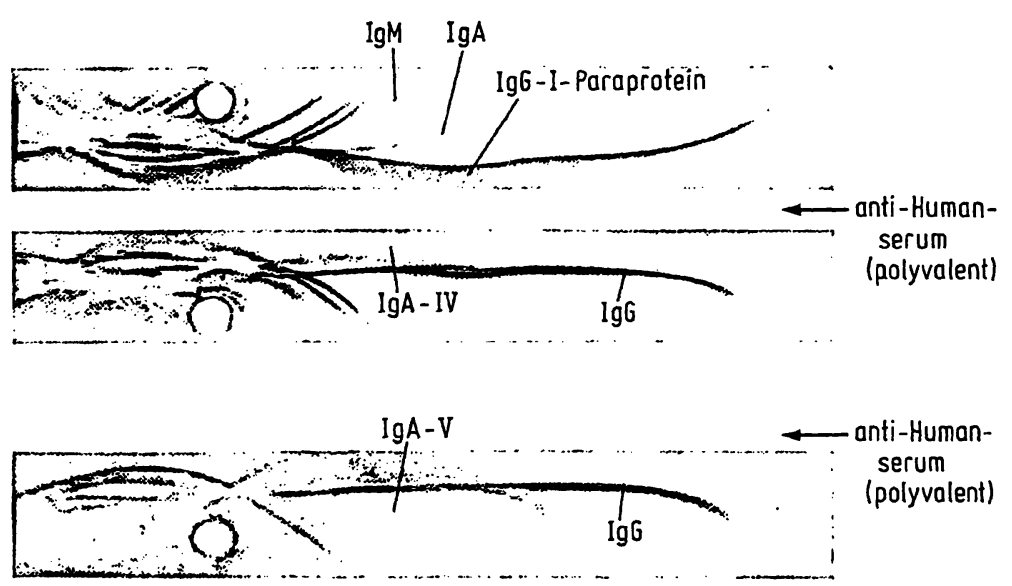

Abb. 3

Immunelektrophoretische Einzelbeispiele von IgA-Paraproteinseren im Vergleich zu einem IgG-I-Myelomprotein Ausschnitte des $\beta / \gamma$-Bereiches bzw. des $\alpha_{2} / \beta$-Bereiches

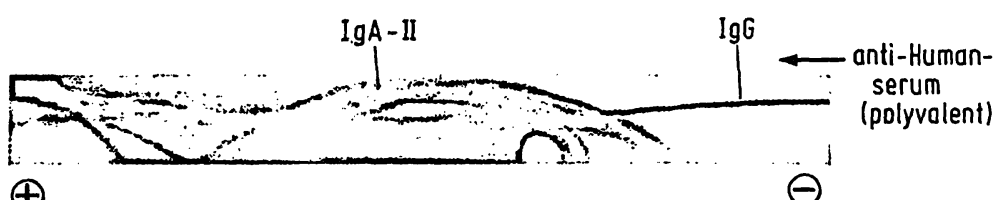




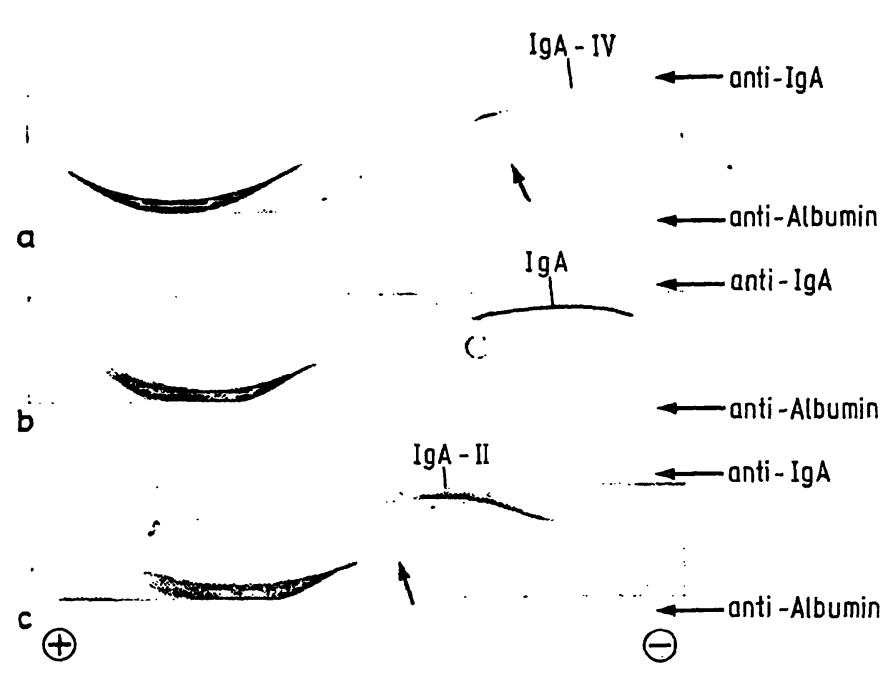

Abb. 4

Immunelektrophoretische Darstellung der Komplexbildung von IgAParaprotein mit Albumin-Molekülen

a) IgA-IV-Paraproteinserum

c) IgA-II-Paraproteinserum

begrenzte, schwach auspräzipitierte Linie, die sich bis in den physiologischen Bereich des IgA erstreckt (vgl. Abb. 2). IgAParaproteine haben außerdem die Eigenschaft, mit Albuminmolekülen Komplexbildungen einzugehen. Dies kann damit bewiesen werden, daß sich bei vergleichender immunelektrophoretischer Analyse eines IgA-Paraprotein-Serums bei Verwendung eines anti-IgA-Serums und eines anti-Albumin-Serums auf der Seite des anti-Albumin-Kanals ein albuminspezifisches Präzipitat an der Stelle des IgA-Paraproteins nachweisen läßt (vgl. Abb. 4). Physiologisches IgA zeigt dieses Phänomen gegenüber Albumin bzw. anti-Albumin nicht!

Bei der Beurteilung der Einzelbefunde des Analysensatzes 1 ist weiterhin darauf zu achten, ob mit Bezug auf die verbliebenen physiologischen Immunglobuline die spezifischen Präzipitate komplett nachweisbar oder ob sie im Sinne eines Antikörpermangels reduziert oder überhaupt nicht mehr darstellbar sind. Ferner sollte das gesamte Liniensystem aus dem Befund mit polyvalentem Antihumanserum auf - semiquantitativ faßbare Besonderheiten durchgemustert werden: Reduzierung oder Fehlen des Prä-Albumins, Verminderung des Albumins, des Transferrins, des Hämopexins sowie Vermehrung des $\alpha_{1}$-Antitrypsins, des sauren $\alpha_{1}$-Glykoproteins, des Coeruloplasmins und auch des Haptoglobins können bei IgA-Paraproteinämien nachweisbar werden.

Bei Beurteilung der Platte 2 interessiert vor allem die Zuordnung des IgA-Paraproteins zu einem der beiden Leichtketten-Typen und/oder die Diagnostik eines BENCE-JONEs-Proteins.

Werden weder $x$ - noch $\lambda$-Leichtketten im Paraprotein-Bereich nachgewiesen, so muß an eine $\mathrm{H} \alpha$-Ketten-Krankheit gedacht werden. In diesem Falle muß wiederum Urin des Patienten mituntersucht werden, da erfahrungsgemäß $\mathrm{H} \alpha$-Ketten nierengängig sind. Außerdem sollte eine Ultrazentrifugen-Analyse des Serums und des Urins vorgenommen werden, um über das Molekulargewicht des fraglichen $\mathrm{H} \alpha$-Proteins indirekt Aufschluß zu bekommen.

\section{Zweidimensionale Doppel-Inumundiffusion (5)}

Diese Technik, die sowohl im Agar- als auch im Agarosegel ausgeführt werden kann, eignet sich im Rahmen der IgA-ParaproteinDiagnostik vor allem zur Auffindung von Äquivalenzzonen, ferner zur IgA-Paraprotein-Diagnostik und Charakterisierung im Urin, in welchem im allgemeinen andere Immunglobuline nicht nachweisbar werden. Immunglobuline der übrigen Ig-Klassen gehen mit anti-Leichtkettenseren ebenfalls Identitätsreaktionen ein und können somit zu Fehlinterpretationen führen. Dasselbe gilt selbstverständlich auch für die Diagnostik einer BENCEJoNEs-Proteinämie und -urie.

\section{Einfacbe, radiale Immundiffusion im Agargel (6)}

Die einfache, radiale Immundiffusions-Technik wird zur quantitativen Bestimmung eines bereits festgestellten IgA-Paraproteins vorgenommen. Sie vermag keinen Aufschluß zu geben über die Differentialdiagnose $z$ wischen IgA-Pataproteinämie und IgAHypergammaglobulinämie, wie sie z. B. bei Leberparenchymprozessen, bei WiskotT-ALDRICH-Syndrom, Erktankungen des broncho-pulmonalen Systems oder bei Enteropathien vorkommen kann. Das quantitative Verhalten des IgA-Pataproteins interessiert aber im Zusammenhang mit Verlaufskontrollen nach Beginn einer bestimmten Therapie oder in Relation zur klinisch faßbaren Prozeßakuität und somit zugleich für prognostische Fragen.

Zur praktischen IgA-Paraprotein-Bestimmung werden bandelsübliche IgA-spezifische Antikörper-Agarplatten ${ }^{3}$ ) verwendet. Es ist darauf zu achten, daß für das IgA-Paraprotein jedes Patientenserums die Äquivalenzzone aufzusuchen ist, d. h. daß bei sehr hohen IgA-Paraprotein-Konzentrationen außer der empfohlenen Serumkonzentration mehrere Verdünnungsstufen mitangesetzt werden müssen und $\mathrm{da} \beta$ selbstverständlich bei der Berechnung der Endkonzentration der jeweilige Verdünnungsfaktor berücksichtigt werden muß.

\section{Eigene Befunde}

Wie eingangs erwähnt, umfaßt das vorliegende Erfahrungsgut 122 Patientenseren mit H-Ketten-spezifischen Paraporteinen der IgA-Klasse. Das sind 19\% von 640 Paraporteinseren der Immunglobulinklassen G, A, M und D. Unter diesen 122 Paraproteinseren fand sich das IgA-Paraprotein $75 \mathrm{mal}$ in $\beta / \gamma_{1}$-Position, $34 \mathrm{mal}$ in $\beta-, 6 \mathrm{mal}$ in $\gamma_{1^{-}}, 5 \mathrm{mal}$ in $\alpha_{2^{-}}$und $2 \mathrm{mal}$ in $\alpha_{2} / \beta$-Stellung (Tab. 1). Diese Differenzierung konnte sowohl in den Celluloseacetatfolien- oder PapierElektrophoresediagrammen (Abb. 1) als auch immunelektrophoretisch (Abb. 2, 3) objektiviert werden.

Ein BENCE-Jones-Protein konnte in 26 Fällen, das sind $21,3 \%$ der IgA-Paraproteinseren bzw. im entsprechenden Urin, nachgewiesen werden (Tab. 2). Über den Nachweis eines sekundären Antikörpermangelsyndroms gibt Tabelle 3 Auskunft.

3) Partigen-Immundiffusionsplatten, Behringwerke AG, Marburg/ Lahn; Immunoplates, Hyland/Travenol International München.

Tab. 1

Prozentualer Anteil von IgA-Paraprotein-Typen von 640 untersuchten Paraproteinseren verschiedener Klassen Darunter: Häufigkeitsverteilung der einzelnen IgA-Paraprotein-Typen unter den beobachteten IgA-Paraproteinseren

\begin{tabular}{cccccccc}
\hline & $\begin{array}{c}\text { Paraprotein- } \\
\text { seren }\end{array}$ & $\begin{array}{c}\text { IgA } \\
\text { Paraproteine }\end{array}$ & $\begin{array}{c}\alpha_{3} \\
\text { IgA-I }\end{array}$ & $\begin{array}{c}\alpha_{2} / \beta \\
\text { IgA-II }\end{array}$ & $\begin{array}{c}\beta \\
\text { IgA-III }\end{array}$ & $\begin{array}{c}\beta / \gamma_{1} \\
\text { IgA-IV }\end{array}$ & $\begin{array}{c}\gamma_{1} \\
\text { IgA-V }\end{array}$ \\
\hline Gesamtzahlen & 640 & 122 & 5 & 2 & 34 & 75 & 6 \\
$\%$ & 100 & 19,0 & 0,8 & 0,3 & 5,3 & 11,7 & 0,9 \\
$\%$ IgA & 100 & 4,1 & 1,6 & 27,9 & 61,5 & 4,9 \\
\hline
\end{tabular}

Z. Klin. Chem. Klin. Biochem. / 11. Jahrg. 1973/ Heft 12 
Tab. 2

Befunde der $x$ - sowie $\lambda$-Eigenschaften der beobachteten IgA-ParaProteine. Darunter: Nachweis und Verteilung von BENCE-JONESMikroproteinen auf die verschiedenen IgA-Paraprotein-Typen

\begin{tabular}{lccccc}
\hline IgA-Typ & $\begin{array}{c}\alpha_{2} \\
\text { IgA-I }\end{array}$ & $\begin{array}{c}\alpha_{2} / \beta \\
\text { IgA-II }\end{array}$ & $\begin{array}{c}\beta \\
\text { IgA-III }\end{array}$ & $\begin{array}{c}\beta / \gamma_{2} \\
\text { IgA-IV }\end{array}$ & $\begin{array}{c}\gamma_{1} \\
\text { IgA-V }\end{array}$ \\
\hline$x$ & 2 & 0 & 21 & 41 & 6 \\
$\lambda$ & 3 & 2 & 13 & 34 & 0 \\
$\begin{array}{l}\text { BENCE- } \\
\text { JONES } x\end{array}$ & 2 & 0 & 6 & 9 & 0 \\
BENCE- & 0 & 1 & 4 & 4 & 0 \\
JONES $\lambda$ & & & & & \\
\hline
\end{tabular}

Eine Komplexbildung des IgA-Paraproteins mit Albuminmolekülen wurde bei insgesamt 18 IgA-Paraproteinseren, darunter drei IgA-Paraproteine in $\alpha_{2}-$ Stellung, geprüft und für bestätigt gefunden (Abb. 4a, c). Physiologisches IgA zeigt dieses Phänomen nicht (Abb. 4b).

Die Untersuchung der L-Ketten-Merkmale ergab in 70 Fällen, das sind $57,4 \%$, den $x$-Typ, in 52 Fällen, das sind $42,6 \%$, den $\lambda$-Typ (Tab. 2).

\section{Diskussion}

Die Beobachtungstatsache, daß IgA-Paraproteine in elektrophoretisch unterschiedlichen Positionen vorkommen können, ist seit Einführung immunologischer Untersuchungstechniken in das klinisch-chemische Routinelaboratorium hinreichend bekannt (4, 6-14). Kunkel und Prendergast (15) sowie Vaerman und Herenrans (16) haben darüber hinaus auch im Serum von Gesunden zwei IgA-Unterklassen, IgA 1 und $\operatorname{IgA} 2$, nachgewiesen, die quantitativ in einem Verhältnis von $1(\operatorname{IgA} 2) \mathrm{zu} 9(\operatorname{IgA} 1)$ vorkommen. $\operatorname{IgA} 2$ unterscheidet sich von $\operatorname{IgA} 1$ durch die biochemisch erwiesene Besonderheit, daß seine H-Ketten zufolge fehlender Cysteinylreste zu den L-Ketten keine Disulfidbrücken besitzen (17). Wahrscheinlich entspricht IgA 1 dem hier beschriebenen Typ IgA-IV, IgA 2 dem Typ IgA-İ. Grey (18) diskutiert eine dritte IgA-Subklasse.

Die eigenen Befunde zeigen, $\mathrm{da} B$ IgA-Paraproteine in fünf verschiedenen, elektrophoretisch definierbaren Positionen vorkommen können. Die Paraproteinphänomene waren jeweils einwandfrei reproduzierbar. Bei Vergleich der immunelektrophoretischen Ergebnisse mit denjenigen der einfachen Elektrophorese auf Celluloseacetatfolie oder Papier konnten ebenfalls fünf verschiedene Stellungen des IgA-Paraproteins wahr- genommen werden. Formanalytisch erwies sich das Paraprotein-Präzipitat in $97,5 \%$ der beobachteten Fälle als mittelständiges Antigenüberschußphänomen der IgA-Linie. In 2,5\% (3 Einzelbeobachtungen) konnte eine Verdickung des IgA-Präzipitats im ersten Drittel festgestellt werden, woraus auf - wahrscheinlich clonal abhängige - Inhomogenität dieser IgA-Fraktion geschlossen werden darf.

Die hier gewählte Bezeichnung der Paraprotein-Typen IgA-I bis IgA-V richtet sich nach der Reihenfolge ihrer elektrophoretischen Position von Anode zu Kathode. Die Verwendung römischer Zahlen soll eine Identität mit den chemisch definierbaten Subklassen des IgA, die bekanntlich mit $\operatorname{IgA} 1$ und $\operatorname{IgA} 2$ symbolisiert werden, ausschließen, da entsprechende molekularbiologische Untersuchungen hier nicht vorgenommen worden sind und da sich in einer Reihe von Beispielen Paraproteine von den ihnen zugrundeliegenden Immunglobulinklassen funktionell unterscheiden und nicht selten auch intramolekulare Differenzen aufweisen.

Nach ihrer Häufigkeit sind unter den IgA-Paraproteinen die Typen IgA-IV und IgA-III am meisten vertreten. Die Typen IgA-I und IgA-II kommen relativ selten vor. Es besteht kein Zweifel darüber, daB IgA-I- und IgA-II-Paraproteine den seltenen,$\alpha_{2}$-Myelomproteinen" der älteren Literatur entsprechen $(7,8,11,13$, in 14, 19-21).

Die Frage dessen, ob es sich bei diesen elektrophoretisch und immunelektrophoretisch faßbaren IgA-ParaproteinTypen lediglich um einen elektrophoretischen Polymorphismus oder zugleich um genetisch determinierbare bzw. clonal abhängige Unterschiede handelt, kann aufgrund der vorliegenden Feststellungen allein nicht beantwortet werden.

IgA-Paraproteine in $\alpha_{2}$-Stellung erweisen sich relativ häufig als BENCE-Jones-Mikroproteine (in 8, 13, 14). Im eigenen Untersuchungsgut wurden drei der festgestellten " $\alpha_{2}$ "- bzw. , $\alpha_{2} / \beta_{1}$ "-Paraproteine. immunchemisch näher charakterisiert ${ }^{4}$ ). In zwei von diesen Fällen konnten sowohl im Serum als auch im Urin BENCE-Jones-Mikroproteine aufgedeckt werden.

Das erwähnte und dargestellte Phänomen einer Komplexbildung von IgA-Paraprotein und Albuminmolekülen steht im Einklang mit den ersten Beobachtungen von Heremans (in 8, in 22).

4) Bei den übrigen IgA-Paraproteinen in $\alpha_{2}$-Stellung wurde nur der Urin mit der herkömmlichen BENCE-JONEs-Protein-Nachweisreaktion geprüft.

Tab. 3

Immunelektrophoretische Befunde der Immunglobuline $G$ und $M$ bei den hier analysierten 122 IgA-Paraproteinseren

\begin{tabular}{|c|c|c|c|c|c|c|c|c|c|c|c|c|c|c|c|}
\hline \multirow[t]{2}{*}{$\begin{array}{c}\text { IgA } \\
\text { Paraprotein }\end{array}$} & \multicolumn{3}{|c|}{$\underset{I g A-I}{\alpha_{2}}$} & \multicolumn{3}{|c|}{$\begin{array}{c}\alpha_{2} / \beta \\
\text { IgA-II }\end{array}$} & \multicolumn{3}{|c|}{$\begin{array}{c}\beta \\
\text { IgA-III }\end{array}$} & \multicolumn{3}{|c|}{$\begin{array}{c}\beta / \gamma_{2} \\
\text { IgA-IV }\end{array}$} & \multicolumn{3}{|c|}{$\begin{array}{c}\gamma_{1} \\
\operatorname{IgA}-V\end{array}$} \\
\hline & + & $\downarrow$ & $\varnothing$ & + & $\downarrow$ & $\varnothing$ & + & $\downarrow$ & $\varnothing$ & + & $\downarrow$ & $\varnothing$ & + & $\downarrow$ & $\varnothing$ \\
\hline IgG & - & 5 & - & - & 2 & - & 16 & 18 & - & 19 & 56 & - & - & 6 & - \\
\hline IgM & - & - & 5 & - & - & 2 & 22 & 3 & 3 & 10 & 28 & 37 & - & - & 6 \\
\hline
\end{tabular}


Die übrigen Befunderhebungen an den vorliegenden 122 IgA-Paraproteinseren, wie z. B. die Verteilung der Leichtketten-Typen auf $x$ - und $\lambda$-Eigenschaften, ferner der Nachweis einer BENCE-JoNEs-Mikroproteinämie sowie die Häufigkeit eines partiellen, sekundären Antikörpermangelsyndroms, sollen im Rahmen dieser vorwiegend formanalytischen Betrachtungen nicht diskutiert werden. Diese Befunde haben hier lediglich den Sinn einer Information.

$\mathrm{Zu}$ erwähnen bleibt, daß SCHNEIDER und BeRNDt (13), vorwiegend bei einfacher Elektrophorese, IgA-Paraproteine im Bereich des Albumins bzw. zwischen Albumin und $\alpha_{1}$-Globulin sowie im Bereich der $\alpha_{1}$ Globulinfraktion beschrieben haben. Entsprechende Befunde konnten im vorliegenden Untersuchungsgut nicht objektiviert werden.

Die Heterogenität des IgA tritt in besonders eindrucksvoller Weise bei Trennung eines Serums oder des isolierten IgA im Polyacrylamidgel zutage $(23,24)$. Diese Ergebnisse sind jedoch nicht ohne weiteres mit Befunden der Immunelektrophorese im Agargel und auch nicht mit jenen auf Celluloseacetatfolie oder Papier zu vergleichen.

Klinisch ist bei IgA-Paraproteinämie in erster Linie an einen Morbus KaHLER zu denken. In der einschlägigen Literatur wird darauf hingewiesen, daß IgA-Myelome nicht selten besonders unreife Plasmazellen aufweisen und außerdem, daß sie mit einer malignen Retikulose vergesellschaftet sein oder in eine solche übergehen können (in 14, 19, 20, 21).

Die vorliegende Mitteilung soll als Interpretationshilfe bei der Differentialdiagnose von Paraprotein-Phänomenen nach einfacher sowie nach Immunelektrophorese dienen. Sie stellt zugleich einen Beitrag zur Diagnostik der relativ seltenen , $\alpha_{2}{ }^{\text {" }}$-Paraproteinämien dar.

\section{Literatur}

1. Koнn, J. (1957), a) Biochem. J. 65, 9- b) Clin. Chim. Acta (Amsterdam) 2, 297-303. - 2. GRASSMANN, W., HanNig, K. \& KNEDEL, M. (1951), Deut. Med. Wochenschr. 76, 333-336. 3. Grabar, P. \& Williams, C. A. (1953), Biochim. Biophys. Acta 10, 193-194. - 4. Grabar, P. \& Burtin, P. (1964), Immunelektrophoretische Analyse. Elsevier (Amsterdam). - 5. Ouchterlony, Ö. (1949), Acta Pathol. Microbiol. Scand. 26, 507-515. - 6. Arquembourg, P., Salvaggro, J. E. \& Bickers, J. N. (1970), Primer of Immunoelectrophoresis. S. Karger, BaselMünchen-New York. - 7. Fateh-Moghadan, A. \& Laniberz, R. (1969), Visum 13/6, 141-145. - 8. Heremans, J. F. (1960), Les Globulines Sériques du Systéme Gamma. Leur Nature et Leur Pathologie. Arscia S. A., Bruxelles et Masson \& Cie, Paris. - 9. Görz, H. (1973), Immunologische Plasmaprotein-Diagnostik. W. de Gruyter \& Co. Berlin-New York. - 10. Götz, H., SchelfFarth, F. \& Bargger, E. (1963), Med. Welt (Munich) 51, 2611-2614. 11. Scheiffarth, F., Götz, H. \& Juchelka, L. (1966), Med. Klin. (Munich) 61, 744-750. - 12. Lohss, F. \& HillmaNN, G.
(1953), Z. Naturforsch. B \&, 706-708. - 13. SCHNEIDER, W. \& Bernd, H. (1969), Praktikum und Atlas der Immunelektrophorese, J. F. Lehmanns Verlag, München. - 14. WurrrmanN, F. \& MÄRKI, H. H. (1963), Dysproteinämien und Paraproteinämien Schwabe Co., Basel-Stuttgart. - 15. Kunkel, H. G. \& Prendergast, R. A. (1966), Proc. Soc. Exp. Biol. Med. 122, 910-913. - 16. Vaerman, J. P. \& Heremans, J. F. (1966), Science 153, 647-649. - 17. Grey, H. M., Abed, C. A., YounT, W. J. \& Kunkel, H. G. (1968), J. Exp. Med. 128, 1223-1236. 18. GreY, H. M. (1969), Adv. Immunol. 10, 51-104. - 19. KönIG, E. \& KNeZEvic, M. (1960), Acta Haemat. 23,172-182. 20. WuhrmanN, F., Wunderly, CH. \& WIEdEManN, E. (1948), Schweiz. Med. Wochenschr. 78, 180-182. - 21. WukETrch, St. \& Siegmund, G. (1961), Wien. Klin. Wochenschr. 73, 473 bis 476. - 22. Heremans, J. F. (1963), Ergebn. Inn. Med. Kinderheilk. 20, 169-188. - 23. SChEuERLEN, P. G. (1964), Klin. Wochenschr. 42, 1060-1066. - 24. ScheuerLeN, P. G. (1968), Internist 9, 56-63.
Prof. Dr. Hilde Götz 1 Berlin 19 Spandauer Damm Klinikum Westend 